\title{
Multiclass Brain Tumor Classification using Region Growing based Tumor Segmentation and Ensemble Wavelet Features
}

\author{
Ghazanfar Latif \\ College of Computer Engineering and \\ Sciences, Prince Mohammad bin \\ Fahd University, Saudi Arabia. \\ glatif@pmu.edu.sa
}

\author{
D.N.F. Awang Iskandar \\ Faculty of Computer Science and \\ Information Technology, Universiti \\ Malaysia Sarawak, Malaysia. \\ dnfaiz@unimas.my
}

\author{
Jaafar Alghazo \\ College of Computer Engineering and \\ Sciences, Prince Mohammad bin \\ Fahd University, Saudi Arabia. \\ jghazo@pmu.edu.sa
}

\begin{abstract}
In this research, an automated method is proposed for Brain tumor classification into four different types which is an important step in brain tumor diagnosis. Most of the recent research studies focus on binomial classification of brain MR image into tumorous and non-tumorous images that can be extracted using image segmentation. Further classification of the extracted tumor into various classes is an area that is yet to be explored. In our work, we propose an automated system to classify the segmented tumor into various classes. First, the wavelet features are extracted from all four MRI modalities (Flair, T1, T1c, T2) and an ensemble feature set is generated to perform the binomial classification using Random Forest trees. Next, tumor area is extracted from the classified tumorous images by using region growing image segmentation algorithm. In the final phase, wavelet features are extracted from the segmented parts and classification is performed for various tumor types (Necrosis, Edema, Enhancing and NonEnhancing). The experiments are performed on 35 cases including 14 Low-Grade Glioma (LGG) and 21 High-Grade Glioma with total $21,700 \mathrm{MR}$ images. An average accuracy of $94.33 \%$ for binomial MR image classification and $96.08 \%$ for multiclass tumor classification is achieved.
\end{abstract}

\section{CCS Concepts}

- Computing methodologies Classification and regression trees - Computing methodologies Neural networks

\section{Keywords}

Brain MRI; Region Growing; Ensemble Wavelet Features; Tumor Segmentation; Multi-class Tumor Classification.

\section{INTRODUCTION}

In human brain, there are tissues known as "Glial" which are supportive tissues and keep the brain neurons in place and intact for functioning well. Any tumor which is caused by such tissues is

Permission to make digital or hard copies of all or part of this work for personal or classroom use is granted without fee provided that copies are not made or distributed for profit or commercial advantage and that copies bear this notice and the full citation on the first page. Copyrights for components of this work owned by others than ACM must be honored. Abstracting with credit is permitted. To copy otherwise, or republish, to post on servers or to redistribute to lists, requires prior specific permission and/or a fee. Request permissions from Permissions@acm.org.

ICCBD '18, September 8-10, 2018, Charleston, SC, USA

(C) 2018 Association for Computing Machinery.

ACM ISBN 978-1-4503-6540-6/18/09...\$15.00

DOI: https://doi.org/10.1145/3277104.3278311 termed as "Glioma". This tumor mainly occurs in brain and spinal cord. The type of glioma tumor depends on the nature of glial cells involved. Gliomas has different types and each type have specific traits which could affect the brain function and could be life threatening [1]. Therefore it is necessary to classify the glioma type and location so that timely treatment and prognosis which may include chemotherapy, surgery or radiation therapy [2].

Although extensive research has been conducted in this field, still early diagnosis remained poor. The aggressive form of this disease termed as High Grade Gliomas (HGG) in which the survival rate is two or less number of years and immediate treatment is required [3]. There are Low Grade Gliomas (LGG), with slow growing rate and the life expectancy is several years. That's why the main focus is on these HGG which are heterogamous in nature with irregular shape and boundary but experiments are performed on both HGG and LGG. Furthermore the location and size vary considerably which makes the segmentation task more challenging [4]. This is a really crucial task in order to follow up the treatment of HGG and LGG patient. The manual segmentation of such tumor through MR Imaging (MRI) is still a trusted process, performed by a neuro-radiologist. The MRI process is still preferred over other radiology methods as it is more accurate to locate the tumor cells [5]. But this manual process is very tedious and long, to alleviate such limitations, extensive research performed over automated brain tumor segmentation. Classification methods, which differentiate the tissues on intensity based, are discussed in [6]. Such methods are further categorized in to Supervised and Unsupervised classification methods. Supervised classifications methods need prior knowledge from training data sets and from this learning, algorithms take the decision. Still these training data sets are extensive and require time consuming pre-processing but produce better results. The working principle of such methods depends on imposing spatial coherence [7] or only knowledge regarding feature specification is required to perform the segmentation [8].

The tumor is classified in to different classes after segmentation. Different classification methods are discussed in [9], e.g. MultiLayer Perceptron (MLP), Support Vector Machines (SVM), Radial Basis Function (RBF) and Random Forest (RF) Tree. These methods perform the classification by incorporating different tumor features. Most commonly used methods for feature extraction are Discrete Wavelet Transform (DWT), Discrete Fourier Transform (DFT) and Discrete Cosine Transform (DCT) [10]. The research article is structured as follows; in section 2, related work is discussed. In section 3, proposed system is described in details and section 4 discusses about experimental 